\title{
The amount of ergonomics and user involvement in 151 design processes
}

\author{
Barbara N.E.Kok ${ }^{\text {a,c }}$, Karin Slegers ${ }^{\mathrm{b}}$ and Peter Vink ${ }^{\mathrm{c}}$ \\ ${ }^{\mathrm{a}}$ Media Arts and Design Academy, KHLim, C-mine 5, 3600 Genk, Belgium ; \\ ${ }^{\mathrm{b}}$ Centre for User Experience Research (CUO), IBBT-K.U.Leuven Future Health Department, Parkstraat 45, 3000 \\ Leuven, Belgium \\ ${ }^{\mathrm{c}}$ Dept. of Industrial Design Engineering, Delft University of Technology, Land bergstraat 15 2628CE, Delft, The \\ Netherlands.
}

\begin{abstract}
Ergonomics, usability and user-centered design are terms that are well known among designers. Yet, products often seem to fail to meet the users' needs, resulting in a gap between expected and experienced usability. To understand the possible causes of this gap the actions taken by the designer during the design process are studied in this paper. This can show whether and how certain actions influence the user-friendliness of the design products. The aim of this research was to understand whether ergonomic principles and methods are included in the design process, whether users are involved in this process and whether the experience of the designer (in ergonomics/user involvement) has an effect on the end product usability. In this study the design processes of 151 tangible products of students in design were analyzed. It showed that in $75 \%$ of the cases some ergonomic principles were applied. User involvement was performed in only $1 / 3$ of the design cases. Hardly any correlation was found between the designers' experience in ergonomic principles and the way they applied it and no correlations were found between the designers' experience in user involvement and the users' involvement in the design process.
\end{abstract}

Keywords: participatory design; applied ergonomics; user involvement; human centered design

\section{Introduction}

Awareness of the importance of ergonomics and the need to create products synchronized with the users' needs has been growing since the midtwentieth century. Many different types of studies on taking user needs into account in product design have been conducted, such as usability studies (e.g. [3] Dumas), [7] ergonomic research, which started already in the second World War and Human-Centred research (e.g.[9] IHCD). These studies include several approaches, amongst others user-centred design (e.g. Nielsen [11]), human-centred design (e.g. Lee [10]), participatory design (e.g. [6] Haines et al.), design \& emotion (e.g. D\&O [3]) etc. These approaches share the same basic goal: obtaining more user friendly design. Research has also shown the importance of using ergonomic principles and the users' involvement in the design process (e.g. Vink [17]). From this point of view a solid implementa- tion of ergonomic principles, usability and other user-centred methodologies in design would be expected, resulting in user friendly products that meet the users' needs. Yet products often seem to fail to meet the user's needs. Van Kuijk [16] identified a gap between expected and experienced usability in this respect. When buying a product, customers have certain expectations toward the product, which upon use often turn out to be wrong expectations. Moreover, some products are so hard to use that consumers need assistance to use them, or even return or abandon the product. [1, 8, 15]

To understand the possible causes of this gap between expected and experienced usability of products, it might be useful to study the actions taken by the designer during the design process that influence the user-friendliness of a product. Clear knowledge of these actions may help to understand whether certain actions influence the usability of the designed prod- 
ucts or not. It can also clarify which actions are commonly taken by designers and which are not.

The aim of the research described here was to study the use of ergonomic principles and users involvement in the design process. Secondly, we wanted to understand whether the experience with ergonomic principles and methods of the designer has an effect on the use of these principles and methods in the design process. And thirdly, the effect of experience with using methods for users involvement on the actual involvement of users in the design process was analysed.

\section{Method}

In this study, the design processes of 151 products designed by students of the Master Product Design education of the Media Art \& Design-faculty (of the Limburg Catholic University College in Belgium) were studied. For their assignments the students need to write a report about the design process. In this study the design reports of the one hundred and fifty one cases were analysed.

\subsection{Design cases}

These design processes were executed by eighty seven different students. Each student conducted one to six cases. For these assignments, students were given a domain (for example critical design) for the product they had to design as well as certain restrictions (e.g. concerning user group, materials, etc.). An example of such a design assignment is: "Design the ultimate mobile means of communication". [19]

Table 1

Case specifications: gender, study level

\begin{tabular}{|c|c|c|c|c|c|c|c|c|c|}
\hline \multicolumn{10}{|c|}{ Gender } \\
\hline \multicolumn{5}{|c|}{$\hat{0}$} & \multicolumn{5}{|c|}{ q } \\
\hline \multicolumn{3}{|c|}{$\#$} & \multicolumn{2}{|c|}{$\%$} & \multicolumn{3}{|c|}{$\#$} & \multicolumn{2}{|c|}{$\%$} \\
\hline \multicolumn{2}{|r|}{111} & & \multicolumn{2}{|c|}{74} & \multicolumn{3}{|c|}{40} & \multicolumn{2}{|c|}{26} \\
\hline \multicolumn{10}{|c|}{ Study levels } \\
\hline \multicolumn{2}{|c|}{$\begin{array}{c}1^{\text {st }} \\
\text { bach }\end{array}$} & \multicolumn{2}{|c|}{$\begin{array}{c}2^{\text {nd }} \\
\text { bach }\end{array}$} & \multicolumn{2}{|c|}{$\begin{array}{c}3^{\text {rd }} \\
\text { bach }\end{array}$} & \multicolumn{2}{|c|}{$\begin{array}{l}1^{\mathrm{st}} \\
\mathrm{ma}\end{array}$} & \multicolumn{2}{|c|}{$\begin{array}{l}2^{\mathrm{n}} \\
\mathrm{ma}\end{array}$} \\
\hline$\#$ & $\%$ & \# & $\%$ & $\#$ & $\%$ & $\#$ & $\%$ & $\#$ & $\%$ \\
\hline 6 & 4 & 39 & 26 & 67 & 44 & 28 & 19 & 11 & 7 \\
\hline
\end{tabular}

bach: year of bachelor; ma: year of master
All assignments were assessed regarding functionality, the analysis used (e.g. problem analysis user target group, solutions, etc.), the design, technology, innovation and process. The weight of each aspect differed for each assignment and other aspects could be assessed as well. The cases were gathered over five years (2006-07 $\rightarrow$ 2009-10) and the majority (74\%) were from Bachelor students. An overview is given in table 1 .

The one hundred and fifty one cases were retrieved from thirteen different design assignments, the number of cases per assignment vary from two to twenty two.

\subsection{Categories}

The report of every case was analyzed to determine whether and how ergonomic principles were applied and whether and how users were involved. All design process were further analyzed to identify and describe the aspects of ergonomics and user involvement applied in the design process.

The aspects of the design processes studied in this research were divided into two categories: ergonomic and functional study (1) and user involvement (2). The category 'ergonomic and functional study' consisted of consulting ergonomic guidelines and functional analysis. The functional analysis consisted of product function and task analysis (FTA), where all the functions of the product were analysed (which actions need to be done, by whom, etc.); product risk an mistake analysis (RMA), where all possible risk of the product and mistakes that can be made by using the product were analysed; FTA \& RMA by selftesting (TF-RM-ST), where the designer did the analysis by testing the product himself and FTA \& RMA designed product, where the designed product was tested itself.

The category user involvement was divided into: questioning users and/or their companions, (in case the users were for example very small children or have a severe mental disorder; observation; feedback on concepts and/or models, where the participant involved users for testing the concepts and models feedback.

\subsection{Analysis}

The goal of this study was to determine whether the experience of the designer has an effect on the application of ergonomic principles and the involvement of users in the design process. In this paragraph 
some characteristics of the studied group are presented. The level of experience was determined by the study year of the participants. The students have two lesson hours in (product)ergonomics per week during the three bachelor years. The design cases of students in the first year were executed at the end of the first year, the cases in the second year of bachelor were conducted in the first semester and in the first part of the second semester. The participants who executed the cases in the first two years had a low level of experience. The participants who conducted the cases in the third bachelor students had a medium experience in ergonomic principles and the master students had high level of experience.

The level of experience in methods of user involvement was done by studying their curriculum. It appeared that the participants have courses in design methods for user involvement at the end of the second bachelor, the design cases in the second year of bachelor are all executed before these courses began, so they have a low experience in methods for user's involvement", in the third year the participants have courses in design methods six hours a week during half a year, they have a medium level of experience. In het following years there are no more specific courses for user involvement, but they have completed the courses in methods in user involvement, they are considered to have a high level of experience.

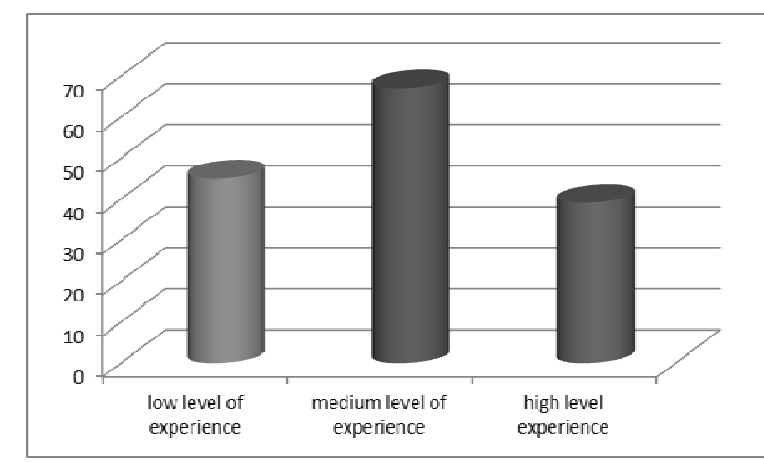

Fig 3: number of cases by level of experience

The level of experience is divided into three groups. The cases performed in the first and second year of bachelor are divided into the group coded a low level of experience, (i.e. low level of experience in ergonomic principles and no experience in methexample when designing for users with a severe mental handicap) or both (1/151). In twenty seven per cent of the cases $(41 / 151)$ observations were performed. The participants asked for feedback on their ods for user involvement). The distribution of experience of the participants is shown in figure 3 .

To determine the aspects of ergonomic and functional study and user involvement, all steps taken during the design process were identified and analysed. Then, for each case an inventory of which of these aspects were used in the design process was made. Thirdly, possible correlations between the participants' experience in ergonomics and the methods used for user involvement were analysed.

In part of the cases one of the supervisors was the teacher in ergonomics $(70 / 151)$ or the teacher in methods for user involvement (26/151), which could affect the results. To trace possible biases, correlations between the supervision of the teacher in ergonomics and the supervision of the teacher in methods for user involvement were analysed as well.

The statistical analysis was done by cross tabulations (in SPSS). The experiences were coded as follows: 1 for a low level of experience, 2 for a medium level and 3 for a high level. When an aspect was performed it was coded 1 if applied (for example users were observed) and coded 0 if it was not applied. The statistical analysis for correlations between experience and application of ergonomic principles or user involvement was performed by crosstabs.

\section{Results}

Table 2 provides an overview of all aspects of ergonomic principles and user involvement identified in this study. In almost every case (145/151) some kind of ergonomic or functional study was conducted. In more than two thirds of the cases (111/151) ergonomic guidelines were consulted. In almost 90 per cent of the cases (132/151) there was a product function \& task analysis (FTA). In 75 per cent $(114 / 151)$ of the cases a product risk \& mistake analysis (RMA) was done. FTA \& RMA by self-testing and FTA \& RMA designed product were executed in less than half of the cases (both 69 out of 151).

User involvement was observed in 42 per cent of the cases (62/151). In one third of the cases (52/151) user involvement was done by questioning users $(36 / 151)$ or their companions $(17 / 151)$. In case it was not possible to question the users (for design by concepts and models in one fifth of the cases (28/151). 


\subsection{Correlations experience \& use of ergonomic principles}

The application of product function and task analysis was the same in all groups, about 90 per cent. There seems to be a difference between the three groups for the risk and mistake: in the group with a low level and the group with a high level of experience it seems that a RMA is performed less often $(76 \% \& 67 \%)$ than in the group with a medium level of experience $(84 \%)$, but the difference is not significant (likelihood ratio $=0,094)$.

The FTA \& RMA of the designed product also seems to be applied more often in the group with medium level of experience (54\% versus $42 \%$ low level \& $31 \%$ high level), but this difference is not significant either (likelihood ratio $=0,065$ ). A significant difference was found for consulting ergonomic guidelines (likelihood ratio $=0,000$ ); this is done more often in by the participants with a low level of experience (100\% versus $63 \%$ low level \& $67 \%$ high level). The FTA \& RMA by self-testing was performed significantly more often in the group with a medium level of experience (54\% versus $42 \%$ low level \& 31 high level; likelihood ratio $=0,004)$. An overview is given in table 3 .
Table 2

application of ergonomic principles and user's involvement

\begin{tabular}{|c|c|c|c|c|}
\hline & $\underset{\mathfrak{Z}}{\stackrel{8}{\mathscr{B}}}$ & $\frac{\overrightarrow{0}}{\stackrel{0}{8}}$ & 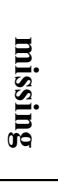 & $\begin{array}{l}20 \\
\stackrel{2}{5} \\
\stackrel{3}{2} \\
\stackrel{2}{2} \\
2\end{array}$ \\
\hline \multicolumn{5}{|l|}{ ergonomic \& functional study } \\
\hline consulting ergonomic guidelines & 111 & 38 & 2 & 74 \\
\hline \multicolumn{5}{|l|}{ functional analysis: } \\
\hline $\begin{array}{l}\text { product function and task analysis } \\
\text { (FTA) }\end{array}$ & 132 & 16 & 3 & 87 \\
\hline $\begin{array}{l}\text { product risk an mistake analysis } \\
\text { (RMA) }\end{array}$ & 114 & 34 & 3 & 75 \\
\hline $\begin{array}{l}\text { FTA \& RMA by self-testing } \\
\text { (TF-RM-ST) }\end{array}$ & 69 & 77 & 5 & 46 \\
\hline $\begin{array}{l}\text { FTA \& RMA designed product } \\
\text { (TF-RM-DP) }\end{array}$ & 67 & 84 & 0 & 44 \\
\hline \multicolumn{5}{|l|}{ user's involvement } \\
\hline $\begin{array}{l}\text { questioning users and/or } \\
\text { Companion }\end{array}$ & 52 & 91 & 8 & 34 \\
\hline Observation & 41 & 108 & 2 & 27 \\
\hline $\begin{array}{l}\text { feedback on concepts and/or } \\
\text { Models }\end{array}$ & 28 & 123 & 0 & 19 \\
\hline
\end{tabular}

Table 3

\begin{tabular}{|c|c|c|c|c|c|c|c|c|c|c|c|c|c|c|}
\hline & & & & & Table 3 & & & & & & & & & \\
\hline & & Experien & e versus & aplicat & ion ergo & nomic prir & ciples & user in & olveme & & & & & \\
\hline & & low level & of experi & ence \# 4 & & medium I & evel of $\epsilon$ & xperienc & \# 67 & high level & of expe & rience \#3 & & \\
\hline & & 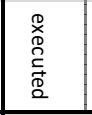 & $\begin{array}{l}\vec{J} \\
\stackrel{+}{0} \\
\text { D }\end{array}$ & 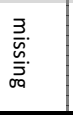 & 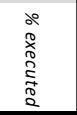 & 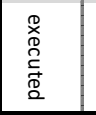 & 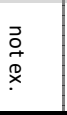 & 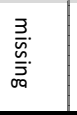 & 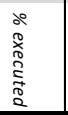 & 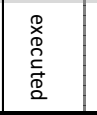 & $\begin{array}{l}\vec{\partial} \\
\stackrel{+}{D} \\
\text { D }\end{array}$ & 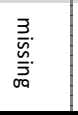 & 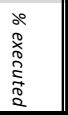 & 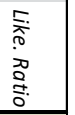 \\
\hline & gonomic functional study & & & & & & & & & & & & & \\
\hline & consutling ergonomic guidelines & 45 & 0 & 0 & 100 & 42 & 25 & 0 & 63 & 26 & 13 & 0 & 67 & 0,000 \\
\hline & functional analysis & & & & & & & & & & & & & \\
\hline & Product function and task analysis (FTA) & 40 & 5 & 0 & 89 & 60 & 6 & 1 & 90 & 34 & 5 & 0 & 87 & 0,832 \\
\hline & product risk an mistake analysis (RMA) & 34 & 11 & 0 & 76 & 56 & 10 & 1 & 84 & 26 & 13 & 0 & 67 & 0,094 \\
\hline & FTA \& RMA by self testing (TF-RM-ST) & 14 & 31 & 0 & 31 & 40 & 24 & 3 & 60 & 17 & 22 & 0 & 44 & 0,004 \\
\hline & FTA \& RMA designed product (TF-RM-DP) & 19 & 26 & 0 & 42 & 36 & 31 & 0 & 54 & 12 & 27 & 0 & 31 & 0,065 \\
\hline & ers involvement & & & & & & & & & & & & & \\
\hline & questioning users and/or companion & 9 & 35 & 1 & 20 & 33 & 29 & 5 & 49 & 11 & 28 & 0 & 28 & 0,001 \\
\hline & observation & 8 & 37 & 0 & 18 & 18 & 49 & 0 & 27 & 16 & 23 & 0 & 41 & 0,060 \\
\hline & feedback on concepts and/or models & 5 & 40 & 0 & 11 & 20 & 47 & 0 & 30 & 3 & 36 & 0 & 8 & 0,005 \\
\hline
\end{tabular}


For the use of ergonomic principles, the application differs int the three groups. The consultation of ergonomic guidelines is lower in the groups a medium and high level of experience. For RMA and FTA \& RMA by self-testing a significant difference was found between the three groups but could not related to the level of experience since the applications higher in the group with a medium level of experience than in the group with low and high level of experience.

Regarding user involvement, significance differences are found between the three groups. For questioning users or their companion and user feedback on the designed product a significant difference was found between the three groups but this could not be related to the level of experience since the application is higher in the group with a medium level of experience than in in the group with a low level and the group with a high level of experience.

\subsection{Effect specialism of the supervisors}

To check whether the results were affected by the fact that the participants were supervised by an ergonomist or specialist in methods for user involvement,

Table 4

Specialism supervisor versus aplication ergonomic principles \& user involvement

\begin{tabular}{|c|c|c|c|c|c|c|c|c|c|c|c|c|c|c|c|c|c|c|c|}
\hline & & & & & \multicolumn{2}{|c|}{ Table 4} & & & & & & & & & & & & & \\
\hline & \multicolumn{19}{|c|}{ Specialism supervisor versus aplication ergonomic principles \& user involvement } \\
\hline & & \multicolumn{4}{|c|}{ pervision by ergonomist \# } & \multicolumn{4}{|c|}{ not supervision by ergon. } & \#81 & \multicolumn{4}{|c|}{$\begin{array}{c}\text { supervision by teacher } \\
\text { meth. in user } \\
\text { involvement } \# 26\end{array}$} & \multicolumn{4}{|c|}{$\begin{array}{c}\text { supervision by teacher } \\
\text { meth. in user } \\
\text { involvement } \# 125\end{array}$} & \\
\hline & & 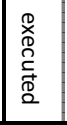 & $\begin{array}{l}\vec{\partial} \\
\stackrel{1}{D} \\
\stackrel{D}{x}\end{array}$ & 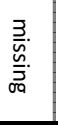 & 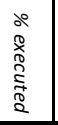 & $\begin{array}{l}\mathscr{R} \\
\stackrel{1}{D} \\
\stackrel{2}{C} \\
\stackrel{+}{D} \\
\stackrel{2}{2}\end{array}$ & $\begin{array}{l}\vec{a} \\
\stackrel{+}{0} \\
\stackrel{D}{x}\end{array}$ & 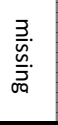 & 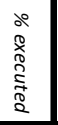 & 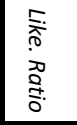 & $\begin{array}{l}\stackrel{\mathbb{R}}{\times} \\
\stackrel{\mathbb{D}}{D} \\
\stackrel{+}{+} \\
\stackrel{\mathbb{D}}{2}\end{array}$ & $\begin{array}{l}\vec{\partial} \\
\stackrel{+}{D} \\
\stackrel{D}{\times}\end{array}$ & 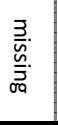 & 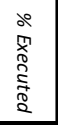 & 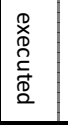 & $\begin{array}{l}\overrightarrow{\vec{O}} \\
\stackrel{+}{\mathbb{D}} \\
\stackrel{x}{0}\end{array}$ & $\begin{array}{c}3 \\
\frac{3}{n} . \\
\frac{\omega}{5} .\end{array}$ & 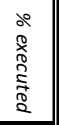 & 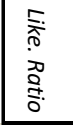 \\
\hline \multicolumn{2}{|c|}{ ergonomic functional study } & & & & & & & & & & & & & & & & & & \\
\hline & consutling ergonomic guidelines & 52 & 18 & 0 & 74 & 61 & 20 & 0 & 75 & 0,885 & 19 & 77 & 0 & 73 & 94 & 31 & 0 & 75 & 0,821 \\
\hline \multicolumn{2}{|r|}{ functional analysis } & & & & & & & & & & & & & & & & & & \\
\hline & Product function and task analysis (FTA) & 63 & 6 & 1 & 90 & 71 & 10 & 0 & 88 & 0,468 & 21 & 5 & 0 & 81 & 113 & 11 & 1 & 90 & 0,147 \\
\hline & product risk an mistake analysis (RMA) & 63 & 6 & 1 & 90 & 53 & 28 & 0 & 65 & 0,000 & 17 & 9 & 0 & 65 & 99 & 25 & 1 & 79 & 0,124 \\
\hline & FTA \& RMA by self testing (TF-RM-ST) & 36 & 31 & 3 & 51 & 35 & 46 & 0 & 43 & 0,202 & 12 & 9 & 0 & 46 & 59 & 63 & 3 & 47 & 0,838 \\
\hline & FTA \& RMA designed product (TF-RM-DP) & 25 & 41 & 0 & 36 & 38 & 43 & 0 & 47 & 0,498 & 15 & 11 & 0 & 58 & 52 & 73 & 0 & 42 & 0,134 \\
\hline \multicolumn{2}{|c|}{ Users involvement } & & & & & & & & & & & & & & & & & & \\
\hline & questioning users and/or companion & 24 & 40 & 6 & 34 & 29 & 52 & 0 & 36 & 0,833 & 17 & 9 & 0 & 65 & 36 & 83 & 6 & 29 & 0,001 \\
\hline & observation & 18 & 52 & 6 & 26 & 24 & 57 & 0 & 30 & 0,592 & 7 & 19 & 0 & 27 & 35 & 90 & 0 & 28 & 0,911 \\
\hline & feedback on concepts and/or models & 18 & 52 & 0 & 26 & 10 & 71 & 0 & 12 & 0,035 & 7 & 19 & 0 & 27 & 21 & 104 & 0 & 17 & 0,245 \\
\hline
\end{tabular}

the possible correlations between the presence of a specialist in the supervisors' group and the application of ergonomic principles or user involvement was analysed.

The supervision of an ergonomist was only found to affect two variables: the application of product risk and mistake analysis (likelihood ratio $=0,000$ ) and asking users for feedback on the design concepts (2D) and tangible models (likelihood ratio $=0,035$ ). The RMA is applied in 90 per cent of the cases, when an ergonomist is part of the supervisors' group and only in 65 percent of the other group. Feedback on concepts and models was requested in twice as often ( $26 \%$ versus $12 \%$ ) cases when there was an ergonomist in the supervisors' group.

The supervision of a specialist in user involvement only affected one variable: questioning users or companions (likelihood ratio $=0,001$ ). Users (or their companions) were questioned twice as often in the group with the specialist in the supervisors' group. (65\% versus $29 \%$ ), which is in line with expectations. The frequencies and correlations are shown in table 4. 


\section{Discussion \& conclusion}

\subsection{Discussion}

The aim of this research was to answer three questions: Are ergonomics principles, methods etc. commonly included in the design process of tangible products? Are users generally involved in this process? And is the designer's experience with ergonomics/user involvement correlated with the application of both ergonomics and user involvement in the design process?

The first question can be answered positively: the use of ergonomic guidelines, the function \& task analysis (FTA) and the risk \& mistake analysis (RMA) was done in 75 per cent of all cases. There was a difference in application in the three groups of different level of experience for consulting guidelines and RMA, but it is still performed in more than 60 per cent of the cases in all groups The function, task, risk \& mistake analysis by self-testing (TF-RM-ST) and function, task, risk \& mistake analysis of the designed product (TF-RM-DP) was performed in a little less than half of the cases. The TF-RM-ST and TF-RM-DP are time consuming, which could explain why these are performed less often. Such a lack of time was often mentioned in the reports of design processes. The academy, the teachers and supervisors find ergonomics very important, which is shown by the high number of hours in ergonomic courses and the high number of assignments supervised by the teacher in ergonomics (7/16 assignments). [19] This could explain why the ergonomic principles are applied in many cases, even though the participants had no experience. Further research is needed on the reasons why some ergonomic principles are applied less often as well as the effect of the application ergonomic principles on the quality of the designed product. In our case we discussed time consumption as one of the reasons, but other reasons could play a role as well; for example Implementing the ergonomic principles could strongly influence the designed shapes, (this is sometimes mentioned in the design reports of the participants).

Concerning the question: "Are users generally involved in this process?" it looks like user involvement was less widely performed in the design processes (only in one third of the cases) than the ergonomic principles. This was unexpected since user involvement is important for the academy [19], which is shown by the many course hours in methods for user involvement. The difference between the performance of ergonomic principles and user involvement could be explained in several ways: involving users in the design process requires more effort from designers than applying ergonomics; ergonomics and functional studies are widely published, more accessible and more published in the participants' native language (e.g. Eger [5], Dirken [2], Voskamp[18]). User involvement also requires a certain amount of time (preparations, making appointments, etc.). The participants often complain about the limited time for their assignments. [Oijevaar [13] also stated in his research that one of the causes of the lack of users involvement is a result of time limits. The lack of time could also explain why user involvement was mainly done by questioning, (1/3), and less by observation (1/4) and even less by feedback on concepts and tangible models $(1 / 10$, see table 2). Questioning was the least time consuming and the easiest way to involve users. Further research is needed on the reasons why users are often not involved as well as the effect of user involvement on the quality of the designed product.

Concerning the correlations, positive correlations were expected between the participants' experience and the application of ergonomics and user involvement.

For the use of ergonomic principles the hypothesis that experience influences the application is only partially true. Negative correlations were found between the experience in ergonomics and consulting ergonomic guidelines. Contrary to the expectations a negative correlation was found suggesting that the more ergonomic experience designers had, the less they consulted ergonomic guidelines in their design processes. A possible explanation could be that the participants throughout the years became very familiar with the guidelines and have less need to consult those guidelines. In the reports written by the participants with a low level of experience it was often mentioned that these guidelines were a good support during the process, while this is hardly ever mentioned in the reports of the other two groups.

For the risk \& mistake analysis (RMA) and function, task, risk \& mistake analysis by self-testing (TF-RM-ST) a significant difference was found between the three groups which cannot be explained by the level of experience since the application was more frequent in the group with a medium level of experience and less frequent in the group with a high level of experience. The difference between the groups may be partially be explained by the effect of 
the composition of supervisors. Positive correlations were found for RMA if there was an ergonomist in the group of supervisors. In more than sixty per cent of the cases in the low and medium level of experience groups were supervised by an ergonomist (see table 5). The fact, that in spite of the high number of cases with supervision by an ergonomist, in only one third of the cases in the low experience level group a TF-RM-ST was conducted suggests that the performance of TF-RM-ST was positively influenced by both experience and the supervision of an ergonomist, but further research is needed to confirm this possible correlation. More research is also needed to determine the factors that influence the application of ergonomic principles in the design process.

Regarding the user involvement, the hypothesis that experience with user involvement influences the decision to involve users was not supported by this study. Significant differences were found between the three groups for questioning users or their companion and user feedback on the designed product. The number of cases involving users was higher in the group with a medium level of experience and lower in the group with a high level of experience. The participants of the group with a medium level of experience had simultaneous with the design assignments the courses in methods for user involvement, through which they paid more attention to user involvement. The participants of the medium experience level group often described importance of user involvement in their reports (in the conclusion). Another possible explanation is the expertise of the supervisors. A positive correlation was found for questioning users (or their companions) and the presence of the teacher in user involvement in the group of supervisors. One third of the cases of the group of medium level of experience was supervised by the teacher in user involvement, (none of the cases in the other two groups were supervised by the teacher in user involvement). The higher number of cases in which users were asked for feedback on the design concepts (2D) and tangible models in the group with a medium level of experience might be explained by both the level of experience and the supervision of an ergonomist, (analogous to the high number of TFRM-ST in the medium level group), further research is needed to confirm this.

There was a positive correlation between the supervision of an ergonomist and asking users for feedback on the design concepts (2D) and tangible models. More research is needed to determine the factors that influence the user involvement in the design process.
Although this study was executed with students' design cases instead of cases of professional designers, the analysis of these cases provide a unique opportunity to study elements of the design processes in a rather large amount of cases. Especially, since it is difficult to obtain extensive reports on the design processes of professional designers. In addition, design student cases are representative of the way young professionals work because young designers apply the design techniques and methods they learned during their education. General conclusions cannot be made from this study. Since ergonomics and user involvement are important in this academy, it is possible that the participants apply more ergonomic principles and user involvement than professionals or design students from other academies or universities do. Therefor further research is needed in other academies and in the field to come to more general conclusions.

Another interesting question is whether all the efforts put into ergonomics and usability in the design process really pay off. Are products with attention for ergonomics experienced better and is the quality of these products better, Further research in this are is highly recommended.

Table 5

Specialism supervisors in different level groups

\begin{tabular}{|c|c|c|}
\hline Supervisied by specialist in: & |ergonomics & User involvement \\
\hline number & 70 & 26 \\
\hline \multicolumn{3}{|l|}{ low level of experience } \\
\hline executed & 29 & 0 \\
\hline not executed & 16 & 45 \\
\hline missing & 0 & 0 \\
\hline$\%$ executed & 64 & 0 \\
\hline \multicolumn{3}{|l|}{ medium level of experience } \\
\hline executed & 41 & 26 \\
\hline not executed & 26 & 41 \\
\hline missing & 0 & 0 \\
\hline$\%$ executed & 61 & 39 \\
\hline \multicolumn{3}{|l|}{ high level of experience } \\
\hline executed & 0 & 0 \\
\hline not executed & 39 & 39 \\
\hline missing & 0 & 0 \\
\hline$\%$ executed & 0 & 0 \\
\hline Likelihood Ratio & 0,000 & 0,000 \\
\hline
\end{tabular}




\subsection{Conclusion}

The general conclusion is that some ergonomic principles (using guidelines; function \& task analysis and risk and mistake analysis) are widely implemented in the design process. Other ergonomic principles (such as: function, task, risk \& mistake analysis by self-testing and function, task, risk \& mistake analysis of the designed product) are only performed in a less than half of the cases and user involvement is only performed in less than one third of the cases. Research about how education can increase the performance of these ergonomic principles and user involvement is needed. This is important for education so they can adjust the educational program.

Further research is needed on the effect of the implementation of ergonomic principles in the design process on the usability and the user's experience of the designed products $[2,5,18]$. The performance of ergonomic principles should result in more user friendly products, that's the aim of (product) ergonomics. Analogous the effect of user involvement on usability and the user's experience of the designed products should be studied. Many researchers [11, 14] have stated that user involvement in the design process is essential to achieve user friendly design, so it is interesting to find out if the user involvement actually improves the users' experience of the product.

\section{References}

[1] den Ouden, E., Yuan, L., Sonnemans, P.J. M., Brombacher, A.C. (2006) Quality and Reliability Problems from a Consumer's Perspective: an Increasing Problem Overlooked by Businesses, Qual. Reliab. Engng. Int. 2006; vol 22:821-838.

[2] Dirken, H. (1997) Productergonomie: ontwerpen voor gebruikers. Delft, NL, VSSD.

[3] Design and emotion, retrieved 20 sept 2011 from http://www.designandemotion.org.

[4] Dumas, J. The great leap forward: The birth of the usability profession (1988-1993) Journal of usability studies Vol. 2, Issue 2, February 2007, pp. 54-60.

[5] Eger, A., Bonnema, M., Litters, E. , van der Voort, M. (2010) Productontwerpen. Den Haag, Netherlands, Lemma, 2010.
[6] Haines, H., Wilson, J.R. , Vink, P., Koningsveld, E., Validating a framework for participatory ergonomics (the PEF). Ergonomics, Volume 45, Issue 4, 15 March 2002, Pages 309-327.

[7] Harel T., "The History of Ergonomics",EasyPublish, Dec 2009, retrieved may 5, 2011, from: http://www.submityourarticle.com.

[8] Horrigan, J. B. and S. Jones, When Technology Fails. Pew Research Center Publications, 2008.

[9] IHCD, Institute for Human Centered Design, retrieved june 10, 2011, from http://www.adaptenv.org/

[10] Lee, L., Learning Spaces: Human-Centered Design: Guidelines. Educause, 2006.

[11] Nielsen J., Usability 101: introduction in usability. Alertbox, retrieved may 2010, www. useit.com

[12] Norman, D. The Design of Everyday Things., New York, USA, Basic Books, 1986.

[13] Oijevaar, K.J., Jovanovic, M. den Otter, A.F.H.J., User Involvement in the Design Process of Multifunctional Buildings. In Wamelink, H., Prins, M. \& Geraerdts, R. (Eds.), CR09, Changing Roles: New Roles and New Challenges, (pp. 485-495). Delft: University Press, 2009.

[14] Sanders, L., Collective Creativity, 3(August 2001)., LOOP American Institute of Graphic Arts, Virginia Commonwealth University Center for Design Studies, USA.

[15] Steger, T., B. Sprague and D. Douthit, Big Trouble with No Trouble Found: How Consumer Electronics Firms Confront the High Cost of Customer Returns. Accenture Communications \& High tech, Accenture, 2007.

[16] Van Kuijk, J.I., Preijde, E.E. , Toet, E.N., Kanis, H. (2009 ${ }^{\circ}$ Expected versus experienced usability: what you see is not always what you get. IEA 2009: the 17th world congress on ergonomics, August 9-14 2009, Beijing, China, IEA.

[17] Vink P.,Imada A.S., Zink K.J., Defining stakeholder involvement in participatory design processes, Applied Ergonomics 39 (2008) 519-526.

[18] Voskamp P., Basisboek ergonomie, Groningen/houten, the Netherlands, Wolters-Noordhoff, 2008.

[19] Zelfevaluatierapport, Media \& Design Academie 1 july 2010, Genk, Belgium. 